\title{
Humane Robots-from Robots with a Humanoid Body to Robots with an Anthropomorphic Mind
}

\author{
GIULIO SANDINI and ALESSANDRA SCIUTTI, Robotics, Brain and Cognitive Sciences, Istituto
} Italiano di Tecnologia, Genoa, Italy

\begin{abstract}
CCS Concepts: $\bullet$ Human-centered computing $\rightarrow$ Collaborative interaction; HCI theory, concepts and models; $\bullet$ Computing methodologies $\rightarrow$ Cognitive robotics;

Additional Key Words and Phrases: Human-robot interaction, natural collaboration, cognitive robotics, anthropomorphism
\end{abstract}

\section{ACM Reference format:}

Giulio Sandini and Alessandra Sciutti. 2018. Humane Robots-from Robots with a Humanoid Body to Robots with an Anthropomorphic Mind. ACM Trans. Hum.-Robot Interact. 7, 1, Article 7 (May 2018), 4 pages.

https://doi.org/10.1145/3208954

Since long ago, humans have always dreamt (or had nightmares) and speculated about having robots able to behave and relate to them as if they were humans (Asimov 1950; Turing 1950). The current storytelling about robotics from the media reinforces these expectations, implying that robots will be soon among us in the form of autonomous cars, personal-assistant robots, and robot companions. This vision is confirmed by what we see with our eyes: robots performing feats close to the ones we have witnessed in many science-fiction movies, from back-flipping (Dynamics 2017), to executing tai-chi moves (Pucci et al. 2016) or engaged in social activities (Matarić and Scassellati 2016). However, the risk is that we - roboticists, journalists, and public alike - are falling victim to an illusion. The intelligence and interaction abilities we are ascribing to robotic machines might just be "in the eye of the beholder."

Robots are now available in physical forms and can exhibit movements that are getting impressively more human. As a result, our brain, which has evolved to interact and understand humans, is tricked into interpreting their behavior as if it were generated by a human. Similar to classical visual illusions, in which our brain "completes" the real image in front of our eyes with preexisting expectations-for instance, making us perceive as convex or concave pictures that are flat, as in the "hollow-mask illusion" (Gregory 1970)-in human-robot interaction (HRI), we complete the picture by "humanizing" the robot. Intuitively, we expect that a robot that moves well can do so because it has a brain functioning at a very high level, a mind that is somehow modeled on the basis of our own. We then assume that such ability will generalize to other tasks, guided by a brain with goals and intentions that will enable the robot to work naturally with us perhaps in tasks that look much simpler than its gymnastic exercises. It is counterintuitive to realize that a robot so sophisticated to

Authors' addresses: G. Sandini and A. Sciutti, Istituto Italiano di Tecnologia, Robotics, Brain and Cognitive Sciences, Genoa, 16152, Italy; emails: \{giulio.sandini, alessandra.sciutti\}@iit.it.

Permission to make digital or hard copies of part or all of this work for personal or classroom use is granted without fee provided that copies are not made or distributed for profit or commercial advantage and that copies bear this notice and the full citation on the first page. Copyrights for third-party components of this work must be honored. For all other uses, contact the Owner/Author.

2018 Copyright is held by the owner/author(s).

2573-9522/2018/05-ART7

https://doi.org/10.1145/3208954 
be able to do a backflip might very well not be able to understand a simple instruction, such as "pass me that," or to move appropriately in a shared space so as to avoid conflicts of resources or waiting.

Unfortunately, the field of robotics is not yet as advanced as we expect it to be by now, especially pertaining to the interaction of robots with humans.

\section{From Being Users to Collaborators}

Current personal-assistant machines is relatively good at responding to explicit orders or instructions (consider Siri or Alexa). This implies that for a collaboration to happen, we need to issue explicit commands to a system regarding what to do, when to do it, and sometimes even exactly how to do it. As a result, all the effort of establishing the interaction falls on the shoulders of the human partner, which is, in all respects, a user. Conversely, humans base most of their collaborative behavior on unsaid, covert information. The reading of implicit signals embedded in human actions enables the partners to read the mind and the emotions of the other and plays a crucial role in our social decisions (Sciutti et al. 2018; Vignolo et al. 2017). Intuition is the short term for this ability to capture the essence of what the other person really intends or wants. I can realize that you are upset from the tone of your voice, the speed of your walking or some micro-expressions on your face, even if you are asking me in the kindest words where I have been today, and I will tailor my response accordingly. Importantly, this is what allows us also to understand when to interact or interrupt someone else, by "reading" the situation and following a series of unscripted social rules. This intelligent behavior is unfortunately still unknown to our software, as demonstrated by the unwanted notifications of our smart phones, when we have forgotten to turn off the volume.

\section{Beyond Real Time}

Although humans generally believe themselves to be focused on the here and now, the human brain is projected into the future: the brain is used to predicting the future (Berthoz 1997). We continuously imagine our actions and their potential effects, simulating our own movements through internal models that we learn and adapt to changes in our body (Bhat et al. 2016). Thanks to the high similarity among conspecifics, the model of ourselves can represent a good approximation of the model we need to interpret and predict others. Therefore, whenever we execute or observe one action, we are already predicting its future evolution and sensory consequences (Flanagan and Johansson 2003). Even infants understand others' actions in terms of their future goals rather than on the basis of the current movement (Meltzoff 1995). Such social prospection is vital in allowing for timely coordination and collaboration. Current machines are often still blocked in an actionperception loop, trying to compensate for the inherently reactive nature of the resulting behavior with incredibly fast computational speeds. However, humans - with all the sensory-motor delays characterizing human nature - are thinking far into the future, exploiting a very different loop based on prediction and exploration, fueled by motivations and driven by experience.

As a result, robots at this writing are not up to speed with human needs. Society today expects robots to respect at least Asimov's first law: "A robot may not injure a human being or, through inaction, allow a human being to come to harm" (Asimov 1942). In other words, a robot should prospectively understand its and its partners' actions and their future consequences in order to avoid any possible negative outcome. Applying this rule requires the robot to project itself into the future, far beyond the limits of its current experience, and sense which objective and subjective implications its actions will have. Humans can do that, robots not - and who knows for how long?

The key to achieve this is probably to stop illusorily humanizing robots and start making them more humane. A humane robot is a robot considerate of humans, that is, one that maintains a model of humans in order to understand and predict human needs, intentions, and limitations, while being transparent, legible, and predictable in Sciutti et al. (2018). The ultimate robot may not be anthropomorphic, but it needs to have at least an anthropomorphic mind (an anthropomorphic 
imagination, to cite Italo Calvino (Calvino 1988)) to support human understanding and prediction. It needs such a model to reply to the question "how would a human behave in this situation?" This interactive model will need to work for robots with very different embodiments, ranging from humanoids to autonomous cars. The difficulty in replicating such a model is that it operates below the level of our awareness. We cannot explicitly express in words what makes us not trust a person at first sight or how we always predict the right place and time to shake someone's hand. Hence, we need to devise a different method that can tap into these implicit processes, revealing which are the visual - or multisensory - features that stimulate the anthropomorphic imagination, communicating implicit knowledge between partners. In this challenge, a humanoid robot can prove useful, serving as a novel investigatory tool and making actual online, embodied collaboration analyzable with the rigorous methods of cognitive science and neurosciences (Sciutti and Sandini 2017). Working with an "artificial conspecific" as a humanoid, it might be possible to follow the human path and learn others' internal models by learning the robot's own during action execution (Zambelli and Demiris 2017). The physical and sensorial similarity with humans can enable the development of a similar anthropometric representation of similar action capabilities and hence of motor resonance, that is, the possibility for robots and humans to relate to each other motorically (Sciutti et al. 2012). The resulting human model could then be used to build an anthropomorphic mind for robots with different embodiments, making them share a more humane perspective of the world.

To summarize, we believe that the greatest challenges for the future of human-robot interaction are the following: (1) gain intuition, becoming partners rather than just sophisticated tools; (2) think beyond real time; and (3) use an anthropomorphic imagination for human-robot interaction. These challenges cannot be met without an honest and active interaction with other disciplines, going beyond the traditional boundaries that see humanities, arts, and journalism as completely detached from robotics and engineering. Indeed, the arts have been much more effective in capturing what a human is in paintings, poetry, and dance than any robotic platform. HRI has a tradition in its effort to bridge different communities. This effort in the future should become even stronger, to make the illusion of humane robots a new reality.

\section{REFERENCES}

I. Asimov. 1942. Runaround. In Astounding Science Fiction. Street \& Smith, New York, NY.

I. Asimov. 1950. I, Robot. Gnome Press, New York, NY.

A. Berthoz. 1997. Le Sens du Mouvement. Odile Jacob, Paris, France.

A. A. Bhat, V. Mohan, G. Sandini, and P. Morasso. 2016. Humanoid infers Archimedes' principle: Understanding physical relations and object affordances through cumulative learning experiences. fournal of the Royal Society Interface 13, 120 (2016), 20160310.

I. Calvino. 1988. Six Memos for the Next Millennium. Harvard University Press, Cambridge, MA.

B. Dynamics. 2017. Atlas Backflip. https://www.bostondynamics.com/atlas.

J. R. Flanagan and R. S. Johansson. 2003. Action plans used in action observation. Nature 424, 769-771.

R. Gregory. 1970. The Intelligent Eye. Weidenfeld and Nicolson, London, UK.

M. J. Matarić and B. Scassellati. 2016. Socially assistive robotics. In Handbook of Robotics, K. O. Siciliano B. (Ed.). Springer, Cham, Switzerland.

A. N. Meltzoff. 1995. Understanding the intentions of others: Re-enactment of intended acts by 18-month-old children. Dev Psychol 31, 838-850.

D. Pucci, G. Nava, and F. Nori. 2016. Automatic gain tuning of a momentum based balancing controller for humanoid robots. In Proceedings of the IEEE-RAS International Conference on Humanoid Robots. IEEE, 158-164.

A. Sciutti, A. Bisio, F. Nori, G. Metta, L. Fadiga, T. Pozzo, and G. Sandini. 2012. Measuring human-robot interaction through motor resonance. International fournal of Social Robotics 4, 223-234.

A. Sciutti, M. Mara, V. Tagliasco, and G. Sandini. 2018. Humanizing human-robot interaction: On the importance of mutual understanding. IEEE Technology and Society Magazine 37 (2018), 22-29. 
A. Sciutti and G. Sandini. 2017. Interacting with robots to investigate the bases of social interaction. IEEE Transactions on Neural Systems and Rehabilitation Engineering 25, 2295-2304.

A. M. Turing. 1950. Computing Machinery and Intelligence. Mind 59, 433-460.

A. Vignolo, N. Noceti, F. Rea, A. Sciutti, F. Odone, and G. Sandini. 2017. Detecting biological motion for human-robot interaction: A link between perception and action. Frontiers in Robotics and AI 4, 14.

M. Zambelli and Y. Demiris. 2017. Online multimodal ensemble learning using self-learned sensorimotor representations. IEEE Transaction on Cognitive and Developmental Systems 9, 113-126.

Received February 2018; accepted April 2018 